Folia Linguistica Historica XIII/1-2 pp. 93-113

(C) Societas Linguistica Europaea

\title{
SUFFIXREANALYSE UND SYLLABIERUNG. ZUM VERHÄLTNIS VON PHONOLOGISCHER UND MORPHOLOGISCHER SEGMENTIERUNG
}

\author{
PETER EISENBERG
}

\section{Reanalysen}

Die Entstehung von Wortbildungssuffixen kann in zahlreichen Fällen als ein Grammatikalisierungsprozeß des zweiten Bestandteils von Komposita verstanden werden. Über Reihenbildung entwickelt sich eine pivot-Struktur, der zweite Bestandteil wird zum Suffixoid. Form und Bedeutung der Einheit passen sich den kombinatorischen Erfordernissen an. Die grammatischen Grundkategorien bleiben - zumindest im Deutschen - weitgehend erhalten.

Daneben entwickeln sich Wortbildungssuffixe auf vielfältige Weise aus anderen Suffixen, meist allerdings so, da $\beta$ unentscheidbar bleibt, wann von einem neuen Suffix zu sprechen ist. Bei Reanalyseprozessen sollte die Frage noch vergleichsweise leicht beantwortbar sein. Reanalyse liegt ja erst dann vor, wenn eine Verschiebung von Segmentgrenzen nachweisbar ist. Es steht das gesamte Inventar segmenteller Analysen zur Verfügung, um den Stand eines solchen Prozesses zu klären.

Grammatikalisierung einerseits und Reanalyse andererseits können zum selben Ergebnis führen, und das im wörtlichen Sinn. Es scheint im gegenwärtigen Deutsch mindestens einen Fall zu geben, in dem man von einem Suffix spricht, in dem aber zwei Entwicklungen zusammengelaufen sind. Das Adjektivsuffix lich ist ja durch Grammatikalisierung eines Nominalstammes in Substantivkomposita entstanden, aber daneben gibt es eine gemeinsame Wurzel mit dem heutigen ig (Willmanns 1896:465f.; Blatz 1900:683f.). Unter Bezug auf eht, den Vorgänger des heutigen ig, beschreibt Blatz den Vorgang so: "Gern tritt -eht an Stamme auf -el z.B. runzeleht, zwirbeleht, ... woraus sich dann durch analoge Übertragung ein Suffix -licht ... bildet, das besonders an Adjektive antritt, z.B. gelblicht, rötlicht, süßlicht".

Damit ist die Struktur aller Abduktionsvorgänge beschrieben, die die vorliegende Arbeit thematisiert. Es handelt sich grundsätzlich um Abduktionsvorgänge durch analoge Übertragung, und nicht um eine Ver- 
schiebung von Segmentgrenzen, die an der Basiseinheit aufgeht. Mit "\#" als Morphemgrenze und "." als Silbengrenze haben wir als Basis für die Reanalyse Einheiten wie zwirbe.l\#eht. Die Reanalyse abduziert nun eine morphologische Einheit leht (spater lich), aber dies führt nicht zu einer

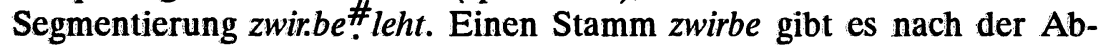
duktion ebenso wenig wie vorher, sondern es gibt nur andere Stämme, an denen leht erscheint. Wie weit die Abduktlon von leht auf zwirbeleht rückwirkt, ist eine der interessanten Fragen. Formal steht jedoch in der Regel außer Frage, daß der Stamm der Basiseinheit nicht in Mitleidenschaft gezogen ist. Plank (1981:74) spricht von "Affix-Reanalyse ohne Reanalyse", was zumindest die Formseite des Vorgangs genau trifft.

Hinweise auf Reanalysevorgänge insbesondere als konsonantische Erweiterungen vokalisch anlautender Suffixe wie im Beispiel eht - leht finden sich in den großen historischen Grammatiken zum Deutschen allenthalben, und auch in den neueren Standardmorphologien (Henzen 1965; Fleischer 1975; Deutsche Wortbildung) ist das Thema keineswegs vernachlässigt. Es paßt aber offenbar schlecht in eine Morphologie, die segmantal-kompositionell zu arbeiten versucht. In der inzwischen doch erheblichen Zahl von Arbeiten zum Deutschen, die im Rahmen der lexikalischen Phononologie vorgelegt wurden, sind mir keine Hinweise begegnet. In jeder Beziehung ertragreich ist Plank (1981). Planks holistische Sicht vermag Reanalyseprozesse als vom Ergebnis her motiviert zu beschreiben, und zwar sowohl was die phonologische wie was die morphologische Seite des Vorgangs betrifft.

Die Zahl der morphologischen Einheiten im gegenwärtigen Deutsch, die durch Reanalyse entstanden sein könnten, dürfte das Dutzend weit übersteigen. Wir thematisieren im Folgenden den Bereich, für den die Lautfolge Schwa + Liquid ([əR], [əl]) in der Abduktionsbasis die entscheidende Rolle spielt. Es geht einmal um den Versuch, das Material zu sichten und wenigstens für einige Einheiten begründete Aussagen über ihren morphologischen Status zu machen (Abschnitt 2). Der dabei immer virulenten Frage, wie morphologische und phonologische Segmentierung aufeinander zu beziehen sind, wird dann am Beispiel der Syllabierung von ler unter Bezug auf Syllabierungshypothesen der lexikalischen Phonologie nachgegangen (Abschnitt 3). Die Erörterung läuft im wesentlichen auf eine Relativierung des Begriffs der Silbengrenze hinaus.

In Abschnitt 2 erschien es einige Male notwendig, den Gesamtbestand an Formen eines bestimmten Typs für das gegenwärtige Deutsche zusammenzustellen. Basis dafür war jeweils Mater 1983, unsystematisch ergänzt aus anderen Quellen und teilweise schon vor der Präsentation bereinigt. Mater führt doch eine große Zahl obsoleter oder anderweitig markierter Wörter auf. Das Datenproblem ist auf diese Weise natürlich 
nicht befriedigend gelöst, gerade angesichts der Tatsache, daß es immer wieder um Aussagen zur Produktivität der betrachteten Affixe geht.

\section{Zur Abduktion von Einheiten mit Schwa + Liquid}

\section{1. erei in Substantivstämmen}

Es finden sich mehrere Typen von Substantiven, die die Vermutung nahelegen, daß es morphologische Einheiten mit der Form erei gibt. Als nicht segmentierbar erweist sich erei in Substantivstämmen wie denen in (1) und (2).

(1) a. Sägerei, Molkerei, Käserei, Riemerei, Zwirnerei, Sklaverei

b. Dieberei, Lumperei, Schlafmützerei, Schelmerei, Schurkerei, Spitzbüberei, Schweinerei, Sauerei

(2) a. Schwelgerei, Tuerei, Schimpferei, Balgerei, Singerei, Rederei

b. Tanzerei, Backerei, Wascherei, Jagerei, Spotterei, Lauferei, Sauferei

Die in (1) sind ihrerseits auf der Basis von substantivstämmen gebildet, die in (2) auf der Basis von Verbstämmen. Der am häufigsten anzutreffende Typ ist der in (2a).

Als phonologische Charakteristika von erei halten wir zweierlei fest. Einmal gehört es zu den Einheiten, die den Hauptakzent des Wortes auf sich ziehen, und zum zweiten hat es stets zwei Silbenkerne. Es gibt kein einziges Vorkommen einer Variante [Rar], auch wenn dies phonotaktisch möglich wäre. Formen wie *Molkrei, *Saurei sind offenbar ausgeschlossen.

Der einfachste Weg zur Behandlung von erei besteht darin, zwei unterschiedliche und voneinander unabhängige Abduktionsvorgänge anzunehmen, nämlich einen denominalen für (1) und einen deverbalen für (2). Beide laufen auf eine Erweiterung des Nominalisierungssuffixes $e i$ hinaus. Betrachten wir zunachst die denominale Abduktion.

Das Suffix ei verbindet sich weitaus überwiegend mit Nomina agentis. Es bildet Substantive mit lokaler Bedeutung ("Ort, an dem die vom Verb bezeichnete Handlung ausgeführt wird"). Die mit Abstand größte Gruppe von Nomina agentis sind ihrerseits Nominalisierungen auf er, so daß es zu einer Ableitungsreihe kommt, die prototypisch enthält backen - Bäcker - Bäckerei, drucken - Drucker - Druckerei, gießen - Gießer - Gießerei, spinnen - Spinner - Spinnerei. Wir stellen damit lediglich fest, da $B$ es solche Ableitungsreihen gibt. Das impliziert weder etwas über die Diachronie der Einheiten noch etwas uber Ableitungsrichtungen. 
Der bei weitem häufigste Kontext für das Auftreten von $e i$ sind Nomina agentis auf $e r$, doch ist $e i$ nicht an diesen Kontext gebunden. Es kommt ebenso vor mit Substantivstämmen anderer Art und führt dann ebenfalls zu solchen mit lokaler Bedeutung (Auskunftei, Detektei, Abtei, Vogtei, vielleicht Türkei, Slowakei).

Der Reanalysevorgang kann nun bei den deverbalen Nomina agentis ansetzen, indem die Morphemfolge $e r+e i$ als eine Einheit erei abduziert wird. Phonologisch spricht nichts gegen eine solche Sicht. erei übernimmt als wesentliche Eigenschaft von el die Akzentuiertheit und als wesentliche Eigenschaft von $e r$ die Silbizität. Das Nominalisierungssuffix $e r$ ist stets silbisch, eine Reduktion auf den Konsonanten ist ausgeschlossen. Selbst in Fällen wie zaubern - Zauberer - Zaubrer mit auslautendem er im Verbstamm bleibt das Nominalisierungssuffix silbisch. Auch in Haplologien wie Zauberin ist $e r$ stets silbisch. Diese Eigenschaft von $e r$ findet sich in der stabilen Zweisilbigkeit von erei wieder, auf die oben hingewiesen wurde. Diese Stabilität von Schwa beruht nicht etwa darauf, daß er hier Suffix ist, sondern sie ist eine Eigenschaft des bestimmten, in Rede stehenden Suffixes. Im Komparationssuffix etwa ist Schwa nicht in der angegebenen Weise stabil, vgl. groß - größer - größerer/größrer (zu den Gründen Eisenberg 1991).

Aus phonologischer Sicht läßt sich also ohne weiteres eine Abduktion von erei ausgehend von er-Nominalisierungen denken, auf der Bedeutungsseite erfaßt man damit aber höchstens die Substantive des Typs 1a. Dieser Typ ist nicht sehr häufig, dazu müssen einige der aufgeführten Substantive als kaum noch gebräuchlich gelten (Riemerei, Zwirnerei), und bei einigen läßt sich zudem auch eine - allerdings mühsame - verbale Herleitung denken (Sägerei, Molkerei). Fraglich ist schließlich, ob Sklaverei die geforderte lokale Bedeutung überhaupt haben kann.

Warum ein Suffix erei auf die beschriebene Weise nicht entstanden ist, wird plausibel, wenn man sich seinen morphologischen Status vor Augen führt. erei in Käserei hatte dieselbe Bedeutung wie $e i$ in Bäckerei und Auskunftei. Es wäre eine - wahrscheinlich prosodisch bedingte -Variante von $e i$. Diese Variante hätte dieselbe Form wie die durchsichtige und höchst produktive Morphemfolge $e r+e i$, aus der sie entstanden sein sollte. Das einfache Zusammenfassen zweier produktiver Suffixe zu einem, das als Variante eines der beiden fungiert, bringt morphologisch keinerlei Vorteile. Es hat aber den Nachteil, daß die Form erei morphologisch mehrdeutig wird. Wir schließen daher aus, daß erei in 1b (Diebe$r e i)$ aus den Suffixen $e r+e i$ entstanden ist, auch wenn dies bisweilen angenommen wird (z.B. Fleischer 1975:135; Henzen 1965:114).

Der zweite Weg zur Abduktion von erei könnte seinen Ausgangspunkt bei Verbstämmen auf er haben. (3) listet die wichtigsten Typen dieser Verben auf. 
(3) a. stöbern, wandern, hindern, schlendern, zaudern, fordern, modern

b. labbern, schlabbern, knabbern, sabbern, bibbern, glibbern, blubbern, gluckern, knattern, schlittern, pladdern, plappern, fleddern stottern, rattern, zittern

c. fiebern, zaubern, hadern, federn, ködern, pudern, opfern, eifern

d. rädern, gliedern, rändern, löchern, geistern

e. albern, säubern ändern, sichern lockern, ermuntern, erheitern

f. mildern, hindern, nähern, schmälern

Die Verbstämme in (3a) sind morphologisch einfach. Gleichlautende Substantivstämme gibt es nicht. Das ist anders in (3c), hier gibt es sowohl den Verbstamm wie den Substantivstamm. In (3d) fungiert eine substantivische Pluralform als Basis. Ähnlich verhalten sich $(3 e, f)$ zueinander. In (3e) ist die Basis ein einfacher Adjektivstamm, in (3f) der Stamm des Komparativs.

Substantive auf erei sind prinzipiell von allen Verbstämmen in (3) bildbar, am durchgängigsten und mit der einheitlichsten Bedeutung aber von denen in (3b). Diese Verben haben eine uniforme Lautstruktur, der gemeinsame Bedeutungsmerkmale entsprechen. Der zweisilbige Stamm aus betonter und unbetonter Silbe hat einen einfachen oder komplexen konsonantischen Anlaut, gefolgt von einem ungespannten Vokal, einem ambisilbischen Plosiv und der charakteristischen Endung ern. Minimalpaare mit Plosiv stimmhaft/stimmlos gibt es nicht, in vielen Fallen sind sogar beide Varianten möglich und gebrauchlich, z.B. [Jlidərn-flitərn], [pladərn-platərn].

Die gemeinsamen Bedeutungsmerkmale der Verben if $(2 b)$ betreffen ein aktionsartliches (iterativ) und ein wertendes (pejorativ) Element. Diese Gemeinsamkeiten der Bedeutung werden in der Literatur durchweg unmittelbar auf die Gemeinsamkeiten der Lautstruktur bezogen, die Formen charakterisiert man als lautmalerisch und schallnachahmend. Charakteristika der morphologischen Struktur spielen danach keine herausragende Rolle. Für alle wird Einfachheit des Stammes unterstellt. Als Alternative käme infrage, ein Verbalsuffix er anzusetzen. Die einigermaßen vollständige Liste von Verben, in denen ein solches Suffix isoliert werden könnte, enthält (4).

(4) a. steigen - steigern, folgen - folgern, rauchen - räuchern, stinken - stänkern 
b. schwappen - schwappern, schruppen -schruppern, klappen - klappern, kletten -klettern, schlecken schleckern, schlinqen - schlingern, lochen - löchern

Die Verben in (4a) weisen nicht die für unsere Gruppe typische Lautstruktur auf. Sie gehören in eines der zahlreichen Muster zur Kausativierung. Die Verben in (4b) haben die geforderte Lautstruktur, und die mit er-Stamm weisen auch die geforderten Bedeutungsmerkmale auf. Bedenkt man zudem, daß mit Sicherheit ein Analogie-effekt von der produktiven Paarbildung mit el (drängen - drängeln, dazu Abschnitt 2.2) ausgeht, dann wird die kategoriale Festlegung auf morphologisch einfach erneut zweifelhaft. Wir wollen dennoch nicht dafür plädieren, die Verbstämme in (3b) als morphologisch komplex anzusehen, noch soll ein Reanalysevorgang für die Abduktion von er erwogen werden. Aber es zeigt sich, daß die Verbstämme in ( $3 b$ ) eine Disposition zur morphologischen Segmentierung haben. Diese Disposition hat die gemeinsame charakteristische Endung er als eine, aber eben nicht als einzige Voraussetzung.

Der Abduktionsprozeß von erei vollzieht sich nach dem Gesagten zweifellos auf der Basis der Verben in (3b). Die abgeleiteten Substantive auf $e i$ wie Schlabberei, Knabberei, Stotterei, Zitterei haben die Bedeutungsmerkmale iterativ und pejorativ, nicht jedoch die lokale Bedeutung der $e i$-Substantive von Agensnominalisierungen wie Bäckerei, Druckerei. Die Abduktion ist vollzogen in der großen Gruppe der Verbstämme aus (2). Auch die in (2b) gehören eindeutig hier her. Formales Kennzeichen des Bezugs auf den Verbstamm ist das Fehlen des Umlauts. Es ergeben sich Paare wie Wascherei - Wäscherei, Backerei - Bäckerei, deren erstes die abduzierte Einheit erei und deren zweites die Suffixfolge $e r+e i$ enthält. In zahlreichen Fällen gibt es einen phonologischen Indikator für den unmittelbaren Bezug auf den Verbstamm nicht. Es liegt dann echte morphologische Ambiguität vor. So kann mit Bildhauerei das wiederholte und vergebliche Bemühen um bildhauerischen Erfolg gemeint sein Struktur (Bildhau)(erei), ebenso wie der Ort, an dem die Bildhauer arbeiten, Struktur (Bildhau)(er)(ei). Ähnlich liegen die Dinge z.B. bei Druckerei, Putzerei, Spinnerei, Packerei, Gießerei Rösterei, Näherei, Färberei und vielen anderen.

(1b) zeigt, daß das vom Verbstamm abduzierte erei auch mit Substantivstämmen verwendet werden kann. Dabei handelt es sich fast durchweg um Nomina agentis. Mit Substantiven dieses semantischen Typs und ere: läßt sich die intendierte Bedeutung realisieren, aber dies ist auch der Typ, auf dem ei in den transparenten Konstruktionen operiert (Bäcker - Bäckerei). Ob dies Zufall ist, muß dahingestellt bleiben.

Laßt sich der morphologische Status von erei in einfacher Weise angeben? In der Literatur finden sich recht unterschiedliche Antworten 
auf diese Frage. Fleischer läßt sie offen, Henzen (1965:113f.) spricht lediglich von einem Suffix, das aus Verschmelzung von $e r+e i$ hervorgegangen sei. In der Deutschen Wortbildung (Bd 3:28; 302) werden $e i$, rei und erei als Varianten desselben Morphems aufgefaßt, also Spinner\#ei, Schurke\#rei, Schelm\#erei. Diese Analyse schließen wir schon wegen des Allomorphs rei aus. Einen Schritt weiter kommt die in der Literatur zur Schwa-Epenthese vertretene Sicht, erei sei ein prosodisch bedingtes Allomorph von ei (Giegerich 1987:455; Hall 1989:826f.). Nach Stämmen mit Betonung auf der Ultima findet sich danach erei (Atm\#erei, Ordn\#erei, Sing\#erei), nach solchen mit Betonung auf der Pänultima findet sich ei (Erläuter\#ei, Meuter\#ei, Erweiter\#ei).

Eine Analyse dieser Art hat ihre Grenze bei Ableitungen von Verben aus (3b). In Schlabberei, Stotterei, Zitterei kann dem Suffix ei keinesfalls dieselbe Bedeutung zugeschrieben werden wie erei in Dieberei, Lumperei. Die Bedeutungsmerkmale iterativ und pejorativ sind ja gerade Merkmale des Stammes. Ebenso ist in Erläuterei, Meuterei, Erweiterei und sämtlichen anderen Ableitungen aus Verbstämmen in (3) ein erei fur die Bedeutungszuweisung erforderlich und nicht nur ein ei. Eine segmentalkompositionelle Sichtweise ist hier auf jeden Fall gegenüber einer zielorientiert-holistischen im Nachteil.

\section{2. $e$ elei in Substantivstämmen, $e l$ in Verbstämmen}

Meist wird elei weitgehend analog zu erei analysiert, Unterschiede sieht man kaum. Die Deutsche Wortbildung (Bd 3:28) weist elei sogar als Variante desselben Morphems aus. Fleischer (1975:135) sieht es wie erei als Pejorativsuffix zur Ableitung von Substantiven aus Substantiven. Für eine solche Auffassung scheinen die Beispiele in (5) zu sprechen.

$$
\begin{aligned}
& \text { Eifersüchtelei, Geheimbündelei, Eigenbrötelei, } \\
& \text { Fremdwörtelei, Handwerkelei, Vernünftelei, } \\
& \text { Deutschtümelei. }
\end{aligned}
$$

Im Unterschied zu erei ist bei elei eine Abduktion als Folge zweier Nominalsuffixe von vornherein ausgeschlossen, ein dem Nominalisierungssuffix $e r$ verbleichbares Suffix $e l$ gibt es nicht. Wenn überhaupt, kann sich elei allein auf der Basis von Verbableitungen etablieren. Folgende Haupttypen von Verben auf $e l$ finden wir vor.

(6) a. meißeln, stapeln, stempein, spachteln, satteln, pinseln, angeln

b. sammeln, gammeln, fummeln, tippeln, humpeln, faseln, nieseln, rieseln 
c. brummen - brummeln, drängen - drängeln; husten hüsteln, lieben - liebeln, spotten - spötteln, streichen streicheln, tanzen - tänzeln, zischen - zischeln, deuten deuteln, heben - hebeln, binden - bandeln, tropfen tröpfeln, häufen - häufeln, äugen - äugeln, lachen - lächeln, haken - häkeln, funken - funkeln, bäumen - bäumeln, kreisen - kreiseln

Die in (6a) haben morphologisch einfache Substantivstämme als Basis, analog zu (3c). Die zweite Gruppe (6b) ist den entsprechenden Verben auf $e r n$ verwandt ('iterativ und schallnachahmend'), jedoch ist ihre lautliche Struktur wie ihre Semantik insgesamt weniger einheitlich. Die dritte Gruppe (6c) gibt es in diesem Umfang bei den Verben auf ern nicht, vgl. (4). Die Erweiterung des Verbstammes um ein silbisches [1], teilweise verbunden mit Umlautung des Stammvokals, restringiert die Bedeutung mit den Merkmalen iterativ und diminuierend. Als morphologisches Muster ist diese Art der Paarbildung produktiv. Aufgrund der spezifischen Bedeutung von el kommt es zu Substantivpaaren wie Brummerei - Brummelei, Drängerei - Drängelei, Spotterei - Spöttelei.

Das Verbalsuffix $e l$ hat - anders als ein Verbalsuffix $e r$ - synchron als etabliert zu gelten. Historisch ist es nach Willmanns (1896:96ff.) bereits im Althochdeutschen von Nominalstämmen auf $l$ abduziert worden. Ein Bezug auf Nominalstämme macht seine heutige Bedeutung zumindest plausibel. Aus Substantivstämmen wie Meißel, Stapel, Stempel, Spachtel (6a) ergeben sich auf offensichtliche Weise Verben mit iterativer Aktionsart. Zum anderen spielt $e l$ auch eine Rolle als Diminutivsuffix, die sogar synchron im Standarddeutschen noch wirksam ist (Stange - Stengel, Busch - Büschel, Bund - Bündel, Sichel, Ferkel, Wichtel, Zettel, Drittel, $M a ̈ d e l)$. Spuren des Abduktionsvorganges von $e l$ sind synchron nicht erkennbar. Auffällig ist allerdings eine phonologische Beschränkung, die für Verben auf $e l$ gilt.

Es scheint so zu sein, daß dem $e l$ im Verbstamm ein Konsonant vorausgehen muß, der nicht höher als [n] in der Sonoritätshierarchie steht. So haben wir wohl Verben mit Nasalen vor dem el (nach Mater z.B. bummeln, baumeln, ähneln, grüneln), nicht aber solche, bei denen dem el ein Liquid oder ein Vokal vorausgeht (*falleln, *murreln, ${ }^{*}$ schreieln, *zieheln). Die meisten dieser Typen sind für Verben auf er sehr gut möglich, vgl. z.B. ballern, trällern, schillern, mauern, scheuern, feiern, wiehern, nähern. Handelt es sich hier nicht um einen Zufall, dann kann man zunächst versuchen, phonologische Gründe für diese Beschränkung geltend zu machen. Die Segmentfolge [OR] wird in der vorliegenden Position standardsprachlich als [e] realisiert, d.h. als offener Vokal. Damit ist selbst nach den offensten Konsonanten und nach geschlossenen Vokalen 
genug Kontur zur Wahrung der Silbigkeit von [əR] vorhanden. Bei [əl] ist das nicht der Fall. Diese Segmentfolge ist eher der Gefahr ausgesetzt, aus artikulatorischen Gründen ihre Silbigkeit zu verlieren.

$\mathrm{Zu}$ einer solchen Entwicklung kann es umso eher kommen, je weniger $e l$ als morphologische Einheit etabliert ist. Anders gesagt: es ist sehr wohl möglich, daß ein Suffix auf phonologische Kontexte beschränkt ist, die seine lautliche Identität sichern. Eine derartige Deutung distributioneller Fakten mag für $e l$ hergeholt erscheinen. Wir werden aber an einem anderen Fall sehen, $\mathrm{da} B$ sie prinzipiell nicht von der Hand $\mathrm{zu}$ weisen ist (Abschnitt 2.4).

Eine abschließende Klärung dieser Frage kann hier nicht erreicht werden. Kaum ein Zweifel besteht aber daran, daß $e l$ als Verbalsuffix etabliert ist, und damit wird sofort der wesentliche Unterschied zwischen elei und erei deutlich. Bei den von den Verben in $6 c$ abgeleiteten Substantiven ergibt sich die Bedeutung rein kompositionell. Ein Substantiv wie Deutelei mit der Segmentfolge Deut\#el\#ei hat eine transparente Struktur. Soweit ich sehe ist dies der einzige Fall des gesamten Formenkreises, für den zweifelsfrei Kompositionalität gegeben ist. In einer auf Kompositionalität gegrundeten Wortbildungslehre wurde ihm wohl eine Schlüsselrolle zugewiesen, auch als Basis fur analoge Bildungen und analoge Interpretationen.

Mit Sicherheit gibt der Typ (6c) die eine analoge Interpretation aller Substantive auf elei ab, unabhangig vom morphologischen Status des el. Substantive wie Meißelei, Stapelei, Stempelei (nach 6a) haben die in Rede stehende Bedeutung ebenso wie Sammelei, Gammelei, Fummelei, Tippelei (nach 6b), obwohl es keinen Anlaß gibt, el hier als morphologische Einheit anzusehen. Die Motiviertheit der Bedeutung geht weiter als aufgrund der morphologischen Struktur zu erwarten wäre.

$\mathrm{Da} \beta$ el als Verbalsuffix etabliert ist, er aber nicht, kann nun u.E. mit einer Reihe von Unterschieden zwischen elei und erei in Verbindung gebracht werden.

1. Zu Substantiven auf elei gibt es regelhaft einen Verbstamm auf $e l$, unabhängig davon, wie die morphologischen Verhältnisse im Einzelnen liegen. Auch bei denominalen Ableitungen wie denen in (5) läßt sich stets ein Verbstamm bilden, vgl. eifersüchteln, fremdwörteln, deutschtümeln. Das ist bei erei-Substantiven etwa aus (1b) anders, vgl. z.B. *diebern, *lumpern, *schlafmützern.

2. Bei den 'lautmalerischen' Verben auf er (3b) ist die phonologische Struktur strikter beschränkt als bei denen auf $e l(6 \mathrm{~b})$. Ein Grund könnte das geringere morphologische Gewicht von er gegenüber el sein.

3. Als intern nicht segmentierbare Einheit ist erei etabliert, elei jedoch nicht. Wie schon bei denominalem er $+e i$ (Typ Bäckerei) drängt sich erneut die Vermutung auf, daß Kompositionalität abduktionshemmend 
ist. Das einfache Zusammenwachsen zweier integrer Suffixe zu einer nicht mehr segmentierbaren Einheit scheint zumindest nicht der gängigste Typ von Reanalyse zu sein.

Es können weitere Gesichtspunkte für den unterschiedlichen morphologischen Status von erei und elei geltend gemacht werden, die nicht unmittelbar auf der oben verfolgten Argumentationslinie liegen, die ihr aber auch nicht widersprechen. Eine phonologische Deutung wird die hohere Sonorität von $[\mathrm{R}]$ gegenüber [1] geltend machen. Höhere Sonorität bedeutet eine stärkere Tendenz zur Silbigkeit und hat etwa nach Auffassung von Giegerich (1987) eine Reihe morphologischer Konsequenzen.

Aus morphologischer Sicht spielt sicherlich eine Rolle, daß er anders als $e l$ ein fast ubiquitäres Suffix ist (Nominalisierung, Adjektiv- und Pronominalflexion, Komparativ, Substantivplural). Als morphologische Einheit geht ihm regelmaßig eine morphologische Grenze voraus, was die Abduktion von erei erleichtern kann. Die Etablierung eines Verbalsuffixes $e r$ wurde darüber hinaus die funktionale Belastung dieser Form weiter erhöhen.

\section{3. ern in Adjektivstämmen}

Ein Suffix ern zur Bildung von Adjektiven auf der Basis von Substantivstämmen (Wachs - wächsern, Blei - bleiern) könnte durch Erweiterung des Suffixes en entstanden sein. Das sehr alte Suffix en (mhd. auch $n$ ) verbindet sich vornehmlich mit Stoffsubstantiven und bildet Adjektive mit der Bedeutung "besteht aus dem betreffenden Stoff", z.B. Gold golden, Erz - erzen. Der Reanalyseprozeß setzt auf die bekannte Weise bei Substantivstämmen auf er wie in Silber - silbern, Leder - ledern an: "Da aber die Endung d'n haufig an Stamme auf $-r$ antrat, z.B. silberd'n (silbern), das in silbern gekürzt werden konnte, ... so sah man nhd. -ern als besondere Ableitungssilbe an und setzte sie auch an Stämme, die nicht auf $-r$ ausgehen." (Blatz 1900:679).

Der Gesamtbestand an ern-Adjektiven ist ungefähr der in (7). Die in (7a) sind auf der Basis von Substantiven auf er gebildet, also (e)nAdjektive.

(7) a. silbern, kupfern, ledern, marmorn

b. gläsern, hölzern, brettern

c. wächern, blechern, tönern, bleiern, stählern, beinern, steinern, gipsern, knöchern

Die in (7b) sind auf der Basis des Pluralstammes gebildet. Dieser Beziehung zum Pluralstamm verdanken die ern-Adjektive in (7c) den Um- 
laut. Wenn immer möglich, wird der Substantivstamm umgelautet. Bei en-Adjektiven ist der Umlaut dagegen ausgeschlossen, obwohl er im Mhd. regelmäßig gebildet wurde (vgl. noch gülden - golden).

Ebensowenig wie ein substantivbildendes erei konnte sich ein adjektivbildendes ern als produktive morphologische Einheit durchsetzen. Die Gründe liegen hier aber wahrscheinlich vor einer Blockade durch die Polyfunktionalität von er. Eine Erweiterung von en zu einem produktiven Suffix ern kann wohl schon deshalb nicht stattfinden, weil en selbst nicht produktiv ist.

Sämtliche konsultierten Morphologien schreiben en als wichtigste die oben angegebene Funktion mit Stoffsubstantiven zu. Bei der ungeheuren Zunahme an Stoffsubstantiven, die das Deutsche gegenwärtig verzeichnet, mußte ein produktives Suffix dieser Art eine wachsende Zahl von Adjektiven derivieren. Der Gesamtbestand an en-Adjektiven ist jedoch etwa der in (8).

$$
\begin{aligned}
& \text { eiben, seiden, golden, erden, stoffen, hanfen, pechen, } \\
& \text { milchen, eschen, buchen, leinen, birken, opalen, metallen, } \\
& \text { wollen, tannen, zinnen, papieren, taften, basalten, } \\
& \text { graniten, samten, eichen, bronzen, erzen }
\end{aligned}
$$

Zahlreiche dieser Adjektive sind obsolet oder aus anderen Gründen ungebräuchlich. Der Gesamtbestand ist in keiner Weise auf den Bestand an Stoffsubstantiven zu beziehen. Es ist fraglich, ob es überhaupt neue Adjektive in dieser Klasse gibt. Zudem ist die Bedeutungszuschreibung "besteht aus dem betreffenden Stoff" offenbar unzureichend. In dieser Bedeutung sind die Adjektive aus (8) beschränkt auf attributiven Gebrauch. Die syntaktischen Möglichkeiten des Normaladjektivs haben sie nur bei übertragener Bedeutung. Es scheint sich insgesamt um eine abgeschlossene, vielleicht schon isoliert und stilistisch markierte Klasse zu handeln. Möglicherweise kann das Suffix en seine alte Rolle zur Bildung denominaler Adjektive nicht mehr spielen, weil en in uberwältigender Zahl bei den partzipialen Adjektiven vorkommt. Wir bemerken noch, $\mathrm{da} \beta$ auch die Adjektive auf ern die eben beschriebene semantaktische Beschränkung aufweisen. Wir haben die eiserne Brücke, aber nicht Die Brücke ist eisern. Dagegen haben wir sowohl sein eiserner Wille als auch Sein Wille ist eisern.

\section{4. ler in Substantivstammen}

Der Übergang von er (Bildung von denominalen und deverbalen Nomina agentis) zu ler ist wohl der bekannteste Fall von Suffixerweiterung im Deutschen überhaupt. Einigkeit besteht darüber, daß der Ausfall von Schwa in Stämmen auf el der Ausgangspunkt für die Abduktion war: 
"mhd. bettel-er ..., sattel-er ... ergaben nach Ausfall des unbetonten - $e$ - in der Mittelsilbe nhd. Bettler, Sattler, ... und die damit verbundene Verschiebung der Sprechsilbengrenze führte zur Segmentierung Bett-ler, Satt-ler ..." (Fleischer 1975:144 f.). Was hier diachron als Ausfall des unbetonten $e$ beschrieben wird, manifestiert sich synchron als Bedingung für die Silbigkeit von [1] im Stammauslaut. Es ist häufig bemerkt worden, daB $[\mathrm{R}]$ im Stammauslaut vor dem jetzt in Rede stehenden Nominalisierungssuffix [OR] fakultativ silbisch oder nichtsilbisch ist (Wanderer -Wandrer, Zauberer - Zaubrer, Eiferer - Eifrer). Das weniger sonore [1] ist hier niemals silbisch (*Angeler - Angler, ${ }^{*}$ Betteler - Bettler, ${ }^{*}$ Satteler - Sattler), und dasselbe gilt natürlich auch für Nasale (*Ordener - Ordner, *Trockener - Trockner). Aus dieser Sicht wäre zuerst eine Abduktion von ner (vgl. dazu 2.5) mit ler zu erwarten, und erst danach die von rer. In der Tat ist nirgendwo von einem Sufflx rer die Rede, während man ler und ner stets in einem Atem nennt. Wir werden jedoch sehen, daß entgegen der aus den Sonoritätsbedingungen ableitbaren Prognose ler als morphologische Einheit sehr viel besser etabliert ist als ner.

Aber zurück zu Fleischers Formulierung, denn sie zeigt schön die Unklarheiten, die über das Verhältnis von phonologischer und morphologischer Segmentierung bestehen. Nicht ganz klar ist zunächst, welche Syllabierung für die mhd. Form betteler angesetzt wird. Wird [ $t]$ bereits als Gelenk angesehen, dann ergibt sich [beț.lər], d.h. es findet keinerlei Verschiebung einer Silbengrenze gegenüber dem Nhd. statt. Der Ausfall von Schwa führt lediglich zum Ausfall der gesamten Silbe [to]. Wird [t] nicht als Gelenk angesehen, dann ergibt sich [be.tə.lər]. Der Ausfall von Schwa führt dann zum Übergang des [t] in die erste Silbe [bet], für die Silbe [loR] ändert sich erneut nichts. Der Ausfall von Schwa hat also keinesfalls etwas mit der Plazierung einer Silbengrenze vor dem [1] zu tun, ganz im Gegenteil. In Wörtern wie Nörgler hat man vor dem Ausfall von Schwa [nœe.gə.lər] und nach dem Ausfall von Schwa [nœe.glər] zumindest als eine Möglichkeit der Syllabierung. Hier gäbe es keine Silbe [loR] mehr, der Abduktionsprozeß wäre jedenfalls erschwert.

Aber nicht einmal in Fällen wie Bettler und Sattler kann man mit Sicherheit von einer Silbe [lor] sprechen. Legt man die in der neueren Literatur verbreitete Auffassung zugrunde, da $\beta$ das [t] hier jedenfalls zur zweiten Silbe gehört, ergibt sich wiederum [tlər] und nicht [ləR] als Silbe. Wir werden auf die Frage der Syllabierung dieser und verwandter Fälle genauer in Abschnitt 3 eingehen. Schon jetzt läßt sich folgendes feststellen. Bei Berücksichtigung der üblichen Syllabierungsregeln gibt es wenig Anlaß, eine Resyllabierung als Ausgangspunkt für die Abduktion von ler als morphologische Einheit anzunehmen.

Der einzige phonologische Gesichtspunkt, der für die Plazierung einer Silbengrenze vor dem [l] in Bettler, Sattler geltend gemacht werden 
könnte, ist die Tatsache, daß [tl] keinen möglichen Silbenanfangsrand in wortinitialer Silbe bildet. Dies wäre jedoch nur dann wirklich begründet, wenn ler nicht lediglich in Einzelfallen, sondern typischerweise nach solchen Konsonanten steht, die wie [t] nicht mit [1] einen Silbenanfangsrand bilden können. Zumindest ein erster Blick auf die Daten spricht dafür, $\mathrm{da} \beta$ es sich so verhält. Um das zu zeigen, betrachten wir den Gesamtbestand der ler-Substantive.

Als morphologische Einheit ist ler dann etabliert, wenn es regelhaft in Substantiven auftritt, die kein [1] im Stammauslaut haben. (9a) listet die Typen von Substantiven, bei denen das der Fall ist, während (9b) diejenigen aufführt, in denen wohlgeformte Silben wie [flər], [glər], [blor] usw. vorkommen.

(9) a. Versöhnler, Kriegsgewinnler, Freischärler, Altsprachler, Tausendfüßler, Zuchthäusler, Abstinenzler, Provinzler, Umstürzler, Hinterwäldler, Ländler, Widerständler, Volkskundler, Weltrekordler, Gewerkschaftler, Arbeitsrechtler, Mittelgewichtler, Sportler, Künstler, Postler, Korbblütler, Häusler

b. Dörfler, Gebirgler, Kunstgewerbler, Sommerfrischler, Tischler, Hinterbänkler

Ist es möglich, daß diese Verteilung Zufall ist? Oder gibt es einfach mehr Substantivstämme mit einem Auslaut gemäß (9a) als solche mit einem Auslaut gemäß (9b)? Mir sind Untersuchungen, die darüber Auskunft geben, nicht bekannt. Immerhin sollte erwähnt werden, daß es für das wohletablierte Adjektivsuffix lich ähnliche Restriktionen gibt wie für ler. Das spricht dafür, daß es zumindest eine Tendenz dieser Suffixe gibt, sich mit Stämmen zu verbinden, von denen sie syllabisch nicht integriert werden können.

$\mathrm{Daß}$ ler neben er als morphologische Einheit etabliert ist, steht außer Zweifel. Unabhängig von möglichen phonologischen Restriktrionen gibt es eine klare Tendenz zur Funktionstrennung insofern ler zu Substantivstämmen, $e r$ dagegen primär zu Verbstämmen tritt. Sieht man ab von wenigen Wörtern wie Kriegsgewinnler und Hinterwäldler, so besteht ein semantischer Unterschied allgemein nicht. Insbesondere hat ler nicht allgemein das pejorative Bedeutungselement, das ihm von Deverbalia wie Nörgler, Fasler, Gammler zugewachsen sein könnte. Einige Sprachkritiker haben ja versucht, ler die pejorative Bedeutung generell anzuhängen, um damit etwa Wissenschafter, Gewerkschafter gegenüber Wissenschaftler, Gewerkschaftler normativ zu favorisieren (dazu Deutsche Wortbildung Bd 2:377).

Zur Funktionstrennung von ler und er gehört nicht nur die Spezialisierung von ler auf die Bildung von Denominalia, sondern auch die von 
er auf Deverbalia. Denominale Nomina agentis auf er gibt es in größerer Zahl nur noch vom Typ Techniker, Musiker, Taktiker, Kritiker, Politiker, Physiker, Kliniker. Angesichts von Chemiker, Zyniker, Historiker, Fanatiker und Skeptiker kann man durchaus eine Suffixerweiterung ker in Erwägung ziehen. Aus phonologischer Sicht ist erwähnungswert, daß der letzte produktive Typ von denominalen er-Substantiven Stamme auf $k$ hat, auf einen Konsonanten also, der sich mit ler zu einer wohlgeformten Silbe kler verbinden könnte.

\section{5. ner in Substantivstämmen}

Die Meinungen darüber, ob ner als morphologische Einheit in gleicher Weise etabliert ist wie ler, sind in der Literatur geteilt. Der Gesamtbestand ist in (10) aufgelistet, wiederum geordnet in Hinsicht auf den Status von ner. Die Substantive in (10a) sind von

a. Öffner, Zeichner, Rechner, Ordner, Eigner, Gegner

b. Pilsner, Hochöfner, Gärtner, Wagner, Schuldner

c. Lügner, Redner, Schaffner, Falkner, Brückner, Glöckner, Pförtner, Rentner

d. Zöllner, Klempner, Bühnenbildner, Partner, Söldner, Klausner

Verben deriviert, in deren Stamm das [n] bereits nichtsilbisch ist. (10b) wird als denominal mit stammbildendem [ən] im Basisstamm angesehen, (10c) ist teils deverbal, tells denominal. [(ə)n] ist hier Flexiv (lügen, reden ... Falken, Brücken ...). Bei vokalisch anlautendem Suffix [ər] wird das [n] in (10b) wie in (10c) nichtsilbisch.

In (10d) schließlich ist keine Basis mit [n] vorhanden, ner könnte als morphologische Einheit segmentiert werden. Aber diese Gruppe von Substantiven ist klein und zudem uneinheitlich. Bei Klempner und Partner bleibt kein Stammorphem, Klausner ist außer Gebrauch. So bleiben nur Bühnenbildner, Zöllner, Söldner.

Vom insgesamt kleinen Bestand an ner-Substantiven ist ein Teil bereits mehr oder weniger außer Gebrauch (Hochöfner, Falkner, Wagner, Klausner, Brückner, Glöckner). Größere Gruppen von Neubildungen sind nicht erkennbar, auch eine spezifische Funktionalität von ner zeigt sich nicht.

Bleibt die Frage, warum sich ler, nicht aber ner als Einheit etablieren konnte, wo doch [n] nach dem Sonoritätskriterium eher zu Nichtsilbigkeit neigen sollte als [1]. Abduktionshemmend für ner könnte sein, daß die Ansatzpunkte für eine Reanalyse vielfältig und damit diffus sind. Es ergeben sich wegen der weiten Verbreitung sowohl von $(e) n$ als auch 
von er so viele verschiedene mögliche ner, daß sich kein Platz für ein Suffix dieser Form findet.

Noch naheliegender ist folgender Grund. Die Abduktion von ler wird mit angestoßen dadurch, daß es zahlreiche Verbstämme auf $e l$ gibt (angeln - Angler). Verbstämme auf en gibt es dagegen nicht (lachenn ${ }^{*}$ Lachner). Die wenigen Verben mit nichtsilbischem [n] im Stamm (10a, c) sind ihrerseits deriviert. Es zählt also nicht die Sonorität, sondern einmal mehr die Distribution des einzelnen Konsonanten im System der morphologischen Einheiten.

\section{Syllabierung}

\section{1. ler und dle Syllabierung wortinterner Konsonantcluster}

$\mathrm{Zu}$ den schwierigsten und in der letzten Zeit meistdiskutierten phonologischen Problemen gehört die Syllabierung mehrsilbiger Wortformen und dabei besonders solcher mit mehreren internuklearen Konsonanten (zum Deutschen zuletzt etwa Hall 1989; Rubach 1990; Yu 1991; Giegerich 1991). Das Verhalten von ler stellt hier einen der für das Deutsche viel behandelten Prototypen dar. Nachdem im vorausgehenden Abschnitt einiges zur Morphologie von ler ausgeführt wurde, ist es möglich, die Syllabierungsprobleme zusammenfassend zu benennen. Das soll im vorliegenden Abschnitt geschehen. Viel mehr ist u.E. nicht möglich, schon weil die Datenprobleme nicht bewältigt sind.

Die Syllabierung internuklearer Konsonanten und Konsonantcluster wird in der neueren Literătur vor allem nach drei Arten von Kriterien vorgenommen.

1. Sonorität und Onsetmaximierung. In Übereinstimmung mit dem universellen Silbenbaugesetz wird grundsätzlich zuerst der Silbenonset maximiert. Das führt dazu, daß in einer Folge von zwei Silben der Onset der zweiten schwer gemacht wird gegenüber der Koda der ersten Silbe, z.B. [hol.priç] und nicht etwa [holp.riç] (holprig), das ja auch zwei wohlgeformte Silben hätte. Die Sonoritätsbedingung erscheint außer als universelles Silbenbaugesetz (Vennemann 1988) vor allem unter der Bezeichnung Sonority Sequencing Principle (Clements 1990). Die Onsetmaximierung wird entweder als Teil des Syllabierungsalgorithmus angesetzt oder ergibt sich aus dem sog. Silbenkontaktgesetz, das das letzte Sonoritätsminimum einer Konsonantfolge der zweiten Silbe zuschlägt (Vennemann 1988).

2. Liegt eine Silbengrenze nicht dort, wo sie nach 1 . zu erwarten wäre, so hat das morphologische Gründe, z.B. [e:.klıç] (eklig, phonologisch) aber [te:k.liç] (täglich, morphologisch; vgl. Vennemann 1982:301f.). Giegerich (1991:7) postuliert, daß bei konsontantlsch anlautendem Suffix 
die Silbengrenze mit der Morphemgrenze zusammenfällt. Damit ist festgestellt, warum die Silbengrenze bei täglich vor dem [1] liegt.

3. Wo die Silbengrenze liegt, hängt davon ab, ob wortintern dieselben Konsonantcluster im Onset zugelassen sind wie wortinitial oder ob es wortintern größere Freiheiten gibt. $\mathrm{Da} B$ umgekehrt der Onset wortinitial unrestringierter ist als wortintern scheint nicht vorzukommen. Es ist vorgeschlagen worden, das Kriterium des 'Law of Initials' zu einem Klassifikationskriterium für Sprachen zu machen (Vennemann 1972, Clements and Keyser 1983). Für das Deutsche ist die Frage erheblich. Von ihrer Beantwortung hängt, wie in Abschnitt 2.4 schon angedeutet wurde, viel für die Syllabierung ab, z.B. [a..tmun] vs. [a:t.muy].

Wir gehen nun die verschiedenen Typen von ler-Substantiven durch, um zu prüfen, welche Syllabierungen sich ergeben und wie sie zu deuten sind. In (11a - d) sind zuerst jeweils zwei Formen aufgeführt, in denen ler nicht als Ganzes morphologisch segmentierbar ist. Rechts stehen Formen, bei denen ler unteilbare morphologische Einheit ist.

(11) a. Nörgler, Jubler - Gebirgler, Gewerbler

b. Gaukler, Stapler - Dörfler

c. Schwindler, Fasler - Ländler, Häusler

d. Tüftler, Kraxler - Postler, Sprachler

Für (11a) ist möglich [nœr.glər, ju:.blər], das steht in Einklang mit den Kriterien 1. und 2. Fur Jubler ist außerdem möglich [ju:p.lər], d.h. hier kann so segmentiert werden, als sei ler morphologische Einheit. Für Nörgler sehe ich auch die Möglichkeit [nœrk.lər], wobei das bekannte Problem auftritt, ob tatsächlich zu [k] auslautverhärtet wird oder ob nlcht besser ein entstimmtes [g] anzusetzen sei. Die Frage ist hier von einiger Bedeutung, weil man argumentieren könnte, nur bei [k] liege wirklich eine Silbe [ləR] vor. Gebirgler und Gewerbler zeigen aber, daß so nicht argumentiert werden kann. Keine der Formen enthält ein stammauslautendes [k], eine realistische Transkription ist vielmehr [gəbIRg.ləR, gəverb.lər]. Das steht in Einklang mit Kriterium 2. Möglich sind jedoch auch stimmhaftes [g] und [b], womit sich ergibt [gəbır.glər, gəver.blor] Insgesamt zeigt sich, daß der morphologische Status von ler keinen Einfluß auf die Syllabierung hat. Nur ein Teil der Syllabierungen läßt sich mit den gesamten Krilterien angemessen erfassen.

Dasselbe gilt für (11b). Neben [gau.klər, fta:.plər] ist ohne weiteres möglich [gauk.ləR, fta:p.ləR]. Letzteres ist mit Kriterium 2 unvereinbar. Bei Dörfler ist die morphologische Segmentierung sicherlich präferiert, aber [dœr.flor] ist keinesfalls ausgeschlossen.

Interessant ist (11c). Möglich sind [fvin.dlər, fa:.zlər] sowie [fvind..lər, fa:s.lər] parallel zu (11a). Dagegen haben wir nicht [len.dlər] und 
[hэI.zlər], sondern nur [lendo.lor] und [hors.lər]. Dieser Unterschied ist aber offenbar nicht primär durch den morphologischen Status von [loR] verursacht, sondern dadurch, daß die Obstruenten [d] und [z] in den Basiseinheiten stammfinal stehen und in dieser Position natürlich entstimmt sind (Land, Haus). Eben dies ist bei schwindeln und faseln nicht möglich, denn hier bleiben [d] und [z] immer stammintern. Dieses Verhalten von Ländler und Häusler widerspricht jeder Theorie, die die Auslautverhärtung 'postlexikalisch' nach Abschluß aller Syllabierungsprozesse ansetzt, wie es fast durchweg in der lexikalischen Phonologie gemacht wird.

(11d) ergibt ebenfalls kein konsistentes Bild. Wie in (11c) bilden der Obstruent mit folgendem [l] keinen möglichen Onset $\left({ }^{*}[t 1],{ }^{*}[\mathbf{s l}],{ }^{*}[\mathrm{xl}]\right)$, Da diese Obstruenten stimmlos sind, werden sie bevorzugt der Koda der ersten Silbe zugeschlagen, was ja in (11c) bei den beiden ersten Formen so nicht möglich war. Die bevorzugte Syllabierung ist hier zweifellos [tyft.ləR, kRaks.lər] und naturlich [post.lər, Jpra:x.lər].

Unsere Aussagen lassen zwei Schlüsse zu. Erstens hat der morphologische Status von ler nicht die Wirkung auf die Syllabierung, die sich aus Kriterium 2 ergeben müßte, und zweitens lassen sich die Daten mit den angegebenen Kriterien nicht konsistent beschreiben. Beschreibbar werden sie erst, wenn bestimmte Syllabierungen ausgeschlossen werden, d.h. wenn man die Daten an die Theorie anpaßt. Dies ist der Weg, der in der Literatur in der Regel beschritten wird.

Nun wird man gegen unser Vorgehen einwenden können, daß die Aussagen zur Syllabierung der Formen in (11) reine Behauptungen seien, deren empirischer Gehalt durch nichts gesichert sei. Dieser Vorhalt muß akzeptiert werden, er besteht zu Recht. Er trifft jedoch auf alle mir bekannten Aussagen zur Syllabierung zu. Genau darum geht es. Es soll festgestellt werden, daß die Aussagen über die Lage der Silbengrenze in der Regel reine Behauptungen des jeweiligen Autors sind.

Einer der seltenen Versuche, die Daten wenigstens zu differenzieren und damit zu einer konsistenteren Anwendung der Syllabierungskriterien zu kommen, findet sich in Giegerich (1991). Giegerich differenziert nach Standardlautung und Umgangslautung im Sinne von Duden (1974). Als Standardlautung gilt ihm, was in Duden (1974) dokumentiert ist. Was nicht Standardlautung ist, ist Umgangslautung.

Umgangslautung ist nach Giegerich "clearly" dadurch charakterisiert, daß als wortinterner Onset nur das zugelassen ist, was auch wortinitlal möglich ist. Deshalb werde hier syllabiert [a:t.lər, vart.nəR] (Adler, Weidner), aber [i.glu, as.drta] (Iglu, Adria). Die Syllabierung der beiden letzten Formen ändert sich in der Standardlautung nicht, wohl aber die der ersten. Nach Duden heißt es [a:dləR, vardnəR]. 
Man kommt auf diese Weise einer empirischen Grundlegung von Aussagen zur Syllabierung keinen Schritt näher, aber immerhin werden die Probleme deutlich, die einer empirischen Fundierung im Wege stehen. Einmal bleibt unklar, woher die Daten zur Umgangslautung kommen. Ein logisches Problem ist, daß Standardlautung und Umgangslautung als disjunkt dargestellt werden. Die Alternative, daß etwa [a:.dləR] auch als Umgangslautung möglich ist, wird nicht erwogen, führt sie doch wieder zu inkonsistenter Datenlage. Und wie sind die Aussagen zur Lage der Silbgengrenze bei Standardlautung fundiert? Wir wissen es nicht. Giegerich (1991:28) teilt ohne Bedenken mit, Standardlautung werde selten verwendet. Viele Sprecher benutzten sie gar nicht, er selbst gehöre zu ihnen. Hinzuzufügen ist, daß auch der Duden $(1974,1990)$ keine expliziten Angaben zur Silbengrenze macht. Wir stehen in diesem Punkt ohne Daten da.

\subsection{Zum Begriff der Silbengrenze}

Eine mögliche Konsequenz der vorausgehenden Darlegungen könnte sein, den Begriff der Silbengrenze so zu fassen, daß Silbengrenzen nicht notwendiger Bestandteil phonologischer Strukturen sind. Insbesondere bei Mehrsilbern wäre es nicht geboten, die wortinternen Silbengrenzen zu spezifizieren. Eine solche vom Hergebrachten doch stark abweichende Sicht muß natürlich im Einzelnen begründet und in ihren Konsequenzen durchdacht werden. Das kann hier nicht geschehen. Es ist lediglich möglich, einige der relevanten Gesichtspunkte zu nennen und damit dem Vorschlag vielleicht eine gewisse Plausibilitat zu verleihen.

Wenn Sprecher gebeten werden, eine Wortform zu syllabieren, dann vollziehen sie eine Aufgabe, die nicht direkt etwas mit ihrem Sprachverhalten zu tun hat. Wo eine Silbengrenze liegt, ist für das Sprechen in den meisten Fällen gleichgültig, es gibt dafür keine einheitlichen phonetischen oder gar phonologischen Indikatoren. Die Frage nach Silbengrenzen ist nicht vergleichbar mit der Frage nach Grammatikalität, sondern viel eher mit Fragen nach kategorialem grammatischem Wissen vom Typ "Ist dies ein Substantiv?" Die auf solche Weise gewonnenen Daten können nicht als Beobachtungsdaten für eine grammatische Analyse gelten.

Was Sprecher auf die Frage nach der Silbengrenze mitteilen, kann alles mögliche sein. Primär dürfte das Bemühen sein, eine Wortform in wohlgeformte Silben aufzuteilen. Die Form [virkuy] (Wirkung) etwa wird syllabiert [VIr.kun], weil dies sie einzige Segmentierung überhaupt ist, die zu wohlgeformten Silben führt. Vokalisch anlautende Silben gibt es im Deutschen nur dann, wenn eine unbetonte Silbe einer vokalisch auslautenden folgt wie in [flis.on, RoI.ıc] (fliehen, reuig). In solchen Fällen 
kann natürlich auch [uy] segmentiert werden, vgl. [tsi.un, bəge:.un] (Ziehung, Begehung). In allen anderen Fällen beginnen sog. vokalisch, anlautende Silben bei Explizitlautung mit einem glottalen Plosiv, jedenfalls soweit der Kernwortschatz betroffen ist. Das führt dazu, daß [uy] in Wirkung nicht segmentiert werden kann.

Vergleichen wir damit die Syllabierung von [VIRklıç] (wirklich), meist angegeben als [virk.lıç]. Phonologisch möglich ist auch [VIr.klıç]. Stehen mehrere Möglichkeiten zur Verfügung, die Form in wohlgeformte Silben zu segmentieren, so wird offenbar die als erste genannt, bei der die phonologische Segmentierung mit der morphologischen zusammenfällt. Dies festzustellen bedeutet etwas anderes als zu behaupten, die Silbengrenze liege vor dem Suffix. Wir wissen gar nicht, ob es eine wortinterne Silbengrenze gibt. Es gibt viele Sprecher, die syllabieren [e:k.lıç] wie [te:k.lıç] (eklig - täglich), aber andere syllabieren [e..klıç], unter ihnen wohl die Mehrzahl der Linguisten, die Kenntnis von Syllabierungstheorien haben.

Wir wollen besser nicht behaupten, daß es Silbengrenzen nicht gibt, denn die vorliegende bescheidene Arbeit ist noch kein Alterswerk. Selbstverstandlich gibt es in vielen Wortformen Indikatoren dafür, welche Segmente zu welcher Silbe gehören. Die Form [vir.kuy] war ein Beispiel. Hier ergibt sich aus der einzigen möglichen Segmentierung in wohlgeformte Silben eine Grenze zwischen zwei Lautsegmenten, die man Silbengrenze nennen kann. Ähnlich bei der Artikulation [ju:p.ləor] für Jubler. Die Auslautverhärtung zeigt, daß das Segment [p] zur ersten Silbe gehört, und damit gibt es wieder eine Segmentgrenze, die man Silbengrenze nennen kann. Wenn konsequent gefragt wird, welche Indikatoren es bei internuklearen Konsonanten für die Zugehörigkeit zur ersten oder zur zweiten Silbe gibt, lassen sich möglicherweise viele Segmentgrenzen ermitteln, die man Silbengrenzen nennen kann. Man redet dabei jedoch über die Frage, welche Segmente zu welcher Silbe gehören können. Das laßt mehrere Lösungen offen und ist wieder etwas anderes, als wenn nach der Silbengrenze gesucht wird.

Günther (1991) erwägt, die Aussagen über die Lage von phonologischen Silbengrenzen als im wesentlichen schriftinduziert anzusehen. Dies ist eine Radikalisierung unserer Thesen über den engen Zusammenhang zwischen graphematischer und - unterstellter - phonologischer Syllabierung (Eisenberg 1989, Butt und Eisenberg 1990). Hat Günther recht, so wäre dies nur ein weiterer Hinweis darauf, daß die Phonologie noch immer viel schriftabhängiger ist als sie meint. Fur die sog. Hochlautung selbst gilt das ebenfalls.

Faßt man 'Silbengrenze' als einen im angedeuteten Sinne abgeleiteten Begriff, so hat das für eine phonologische Theorie u.E. viel weniger Konsequenzen als man vielleicht auf den ersten Blick erwarten sollte. Man 
begibt sich damit ja nicht in die Situation, über Wortformen als silbisch unstrukturiert reden zu müssen. An anderer Stelle haben wir dafür plädiert, stärker vom Begriff der syllabischen Struktur Gebrauch zu machen (Butt und Eisenberg 1990; Eisenberg 1991). Der Begriff der syllabischen Struktur basiert auf dem der Silbenfolge von Wortformen und gestattet es, Eigenschaften von Silben in ihrer Abfolge in Wortformen zu thematisieren. $\mathrm{Zu}$ wissen, wieviele Silben in einer Wortform aufeinander folgen und welche Eigenschaften diese Silben haben, setzt nicht den jetzt in der Literatur verwendeten Begriff von Silbengrenze voraus. In den meisten Fällen ist es zur Ermittlung syllabischer Strukturen nicht einmal notwendig zu wissen, wo die eine Silbe anfängt und die andere aufhört.

Reanalysevorgänge, wie sie der Abduktion von ler zugrunde liegen, stellen einen Extremfall von Interaktion zwischen phonologischen und morphologischen Segmentgrenzen dar. Die Erweiterung eines vokalisch anlautenden Suffixes durch einen Konsonant bedeutet, daß die entstehende Einheit in allen phonologischen Kontexten eine Silbe sein kann. Daß sie damit auch in allen Kontexten eine Silbe sein muß, ist eine Unterstellung. Wir hoffen, gezeigt zu haben, daß es keine empirische Evidenz fur eine solche Unterstellung gibt.

PETER EISENBERG Seminar für Deutche Literatur und Sprache Universität Hannover 3000 HANNOVER BUNDESREPUBLIK DEUTSCHLAND

\section{LITERATURVERZEICHNIS}

Blatz, F.

1900 Neuhochdeutsche Grammatik mit Berücksichtigung der historischen Entwicklung der deutschen Sprache. Erster Band. Einleitung, Lautlehre, Wortlehre (3. Aufl.), (Karlsruhe: J. Lang).

Butt M. und Eisenberg, P.

1989 "Schreibsilbe und Sprechsilbe", Zu einer Theorie der Orthographie, hgg. von C. Stetter (Tübingen: Niemeyer), 33-64.

Clements, George N.

1990 "The role of the sonority cycle in core syllabification", Papers in Laboratory Phonology I. Between Grammar und Physics of Speech, hgg. von John Kingston und Martin Beckman (Cambridge: CUP), 283-333.

Clements, George N. und Samuel J. Keyser

1983 CV-Phonology. A generative theory of the syllable (Cambridge, Mass.: MIT Press).

Duden

1974 Aussprachewörterbuch. Wörterbuch der deutschen Standardsprache (2. Aufl.). (Mannheim: Dudenverlag).

1990 Aussprachewörterbuch. Wörterbuch der deutschen Standardaussprache (3. Aufl.), (Mannheim: Dudenverlag). 
Eisenberg, $\mathbf{P}$.

1989 "Die Schreibsilbe im Deutschen", Schrifisystem und Orthographie, hgg. von Peter Eisenberg and Hartmut Günther (Tübingen: Niemeyer), 57-84.

1991 "Syllabische Struktur und Wortakzent. Prinzipien der Prosodik deutscher Wörter", Zeitschrift für Sprachwissenschaft 10: 37-64.

Eisenberg, P., K.H. Ramers und H. Vater (Hg.)

1991 Reader zur Silbenphonologie des Deutschen. (Tübingen: Narr).

Fleischer, Wolfgang

1975 Wortbildung der deutschen Gegenwartssprache (4. Aufl.), (Tübingen: Niemeyer).

Günther, Hartmut

1991 "Re-rep-lik - Zur Formulierung der Worttrennungsregel im Deutschen", Deutsche Sprache 19.

Giegerich, Heinz

1987 "Zur Schwa-Epenthese im Standarddeutschen", Linguistische Berichte 112: 449-469.

1992 "Onset maximisation in German: the case against resyllabification rules", Silbenphonologie des Deutschen, hgg. von Peter Eiseberg, Karl Heinz Ramers, und Heinz Vater (Tübingen: Narr), 134-171

Hall, Tracy A.

1989 "German syllabification, the velar nasal, and the representation of Schwa", Linguistics 27: 807-842.

Henzen, Walter

1965 Deutsche Wortbildung (3. Aufl.), (Tübingen: Niemeyer)

Mater, Erich

1983 Rückläufiges Wörterbuch der deutschen Gegenwartssprache (Oberursel: Finken).

Plank, F.

1981 Morphologische (Ir-)Regularitäten. Aspekte der Wortstrukturtheorie (Tuibingen: Narr).

Rubach, Jerzy

1990 "Final devoicing and cyclic syllabification in German", Linguistic Inquiny 21: 79-94.

Vennemann, Theo

1972 "On the theory of syllabic phonology", Linguistische Berichte 18: 1-18.

1982 "Zur Silbenstruktur der deutschen Standardsprache", Silben, Segmente, Akzente, hgg. von Theo Vennemann (Tübingen: Niemeyer), 261-305.

1988 Preference Laws for Syllable Structure and the Explanation of Sound Change (Berlin: de Gruyter).

Wellmann, Hans

1975 Deutsche Wortbildung. Typen und Tendenzen der Gegenwartssprache. 2. Haubtteil: Das Substantiv (Düsseldorf: Schwann).

Willmanns, Walter

1896 Deutsche Grammatik. Zweite Abteilung: Wortbildung (Straßburg: Trübner).

Yu, Si-Taek

1992 "Silbeninitiale Cluster und Silbifizierung im Deutschen", Silbenphonologie des Deutschen, hgg. von Peter Eiseberg, Karl Heinz Ramers, und Heinz Vater (Tübingen: Narr), 172-207. 
Bereitgestellt von | Technische Informationsbibliothek Hannover Angemeldet 